REGARDS

SUR LECONOMIE ALLEMANDE

BULLETIN ECONOMIQUE DU CRAC

\section{Regards sur l'économie allemande}

Bulletin économique du CIRAC

$72 \mid 2005$

Varia

\title{
TIC : la RFA bien armée pour la société de l'information
}

Isabelle Bourgeois

\section{OpenEdition}

Journals

Édition électronique

URL : http://journals.openedition.org/rea/287

DOI : $10.4000 /$ rea.287

ISBN : 978-2-8218-0840-9

ISSN : 1965-0787

Éditeur

CIRAC

Édition imprimée

Date de publication : 1 juillet 2005

Pagination : 31-36

ISSN : 1156-8992

Référence électronique

Isabelle Bourgeois, «TIC : la RFA bien armée pour la société de l'information », Regards sur l'économie allemande [En ligne], 72 I juillet 2005, document 4, mis en ligne le 23 juin 2008, consulté le 26 avril 2019. URL : http://journals.openedition.org/rea/287 ; DOI : 10.4000/rea.287 


\title{
TIC : la RFA bien armée pour la société de l'information
}

\author{
Isabelle Bourgeois
}

Contrairement aux apparences, l'Allemagne est en pointe en matière de technologies de l'information et de la communication (TIC). II est vrai qu'on s'y complaît à se mesurer aux leaders mondiaux, dans une logique de benchmarking et d'émulation. II est vrai aussi que, dans certains domaines, l'Allemagne peut mieux faire: en matière de réglementation, d'abord; en matière d'infrastructures à haut débit, ensuite, où elle est en retard par rapport à certains de ses voisins européens. A l'inverse, elle dispose d'un avantage compétitif et d'un potentiel non négligeables si on considère l'effet-levier des TIC à la fois sur la productivité des entreprises et sur les activités de R\&D. Or c'est là une caractéristique structurelle, liée à la structuration sectorielle des activités, au schéma d'innovation et au mode de fonctionnement en réseau des entreprises et acteurs de recherche. S'il est difficile, à la veille d'un changement politique, de prévoir la manière dont le prochain gouvernement mettra en œuvre concrètement, à l'échelle nationale, la stratégie « i2010 " lancée en juin 2005 par la Commission européenne dans le cadre de la stratégie de Lisbonne renouvelée, on peut néanmoins affirmer qu'il agira en priorité sur le cadre (sectoriel et général) réservé aux TIC et qu'il cherchera à développer encore plus les réseaux de partenariat, afin de mieux impliquer les PME dans l'effort de R\&D et de leur permettre d'entrer de plain pied elles aussi dans l'économie du savoir.

"L'Allemagne a le potentiel pour devenir l'un des leaders mondiaux de l'économie de l'information ", à en croire un rapport réalisé par TNS Infratest pour le compte du ministère fédéral de l'Economie et publié en avril 2005. Premier argument : dans le palmarès de compétitivité 2004 du World Economic Forum auquel se réfère le rapport, elle figure au $13^{\mathrm{e}}$ rang - loin derrière le trio de tête composé de la Finlande, des USA et de la Suède, mais juste après la Grande Bretagne $\left(11^{\mathrm{e}}\right)$ et les Pays-Bas $\left(12^{\mathrm{e}}\right)$. Second argument : l'Allemagne était, en 2004, le premier marché ouest-européen des TIC, avec un chiffre d'affaires de 132,7 milliards $€$ qui la place avant la Grande Bretagne (119,3 milliards $€$ ) et la France (93,7 milliards $€$ ), selon les données d'EITO citées par le rapport. Ce marché est en forte croissance, en Allemagne $(+3,3 \%)$ comme en GrandeBretagne $(+4,4 \%)$ et en France $(+3,2 \%)$. Troisième argument : l'effet-levier de technologies transversales qui, s'il est soutenu par quelques mesures ciblées, permettra de surmonter les quelques faiblesses structurelles actuelles.

TIC : Parts de marché mondiales 2003-2005 (en \%)

\begin{tabular}{|lrrr|}
\hline & $\mathbf{2 0 0 5}$ & $\mathbf{2 0 0 4}$ & $\mathbf{2 0 0 3}$ \\
USA & 29,3 & 29,4 & 29,6 \\
Europe de l'ouest (hors RFA) & 23,7 & 23,8 & 23,9 \\
Japon & 14,5 & 14,8 & 15,0 \\
Allemagne & 6,5 & 6,6 & 6,6 \\
PECO ") & 2,0 & 1,9 & 1,8 \\
Autres & 23,7 & 23,5 & 23,1 \\
CA mondial (en milliards $€$ ) & 2043 & 1959 & 1889 \\
\hline
\end{tabular}

Source des données : TNS Infratest Business Intelligence / EITO, mai 2005. *) Bulgarie, Estonie, Hongrie, Lettonie, Lituanie, Pologne, République tchèque, Roumanie, Russie. NB : Total Allemagne 2003: 125 milliards $€$; $2004: 128$ milliards $€ ; 2005: 132$ milliards $€$. 
$19 \%$ des emplois d'Europe dans les TIC allemandes

Une étroite symbiose avec l'industrie

Le rôle moteur de l'automobile et de la construction mécanique

Software : des solutions sur mesure
En termes d'emplois, l'Allemagne est première dans l'UE 15, avec $19 \%$ des 5,9 millions de salariés recensés dans les TIC (Eurostat). Outre-Rhin, les TIC sont la troisième 'branche' d'activités : avec 746000 salariés à la fin 2004, elles figurent après la construction mécanique (effectifs : 1 million) et l'automobile (870 000 ; Destatis, 2005). La majorité des salariés est employée dans le secteur des technologies de l'information où, en 2004, on en recensait 93000 dans la production de hardware et 364000 dans l'ensemble des services liés (dont le développement de software). Et, comme le révèle l'évolution de l'emploi dans les TIC sur les cinq dernières années, le secteur a désormais surmonté la crise déclenchée par l'effondrement de la bulle spéculative (dot-com-crash) de 2000.

Evolution de l'emploi dans les TIC en RFA 2001-2005

\begin{tabular}{|lccccc|}
\hline & $\mathbf{2 0 0 5}{ }^{*}$ & $\mathbf{2 0 0 4}$ & $\mathbf{2 0 0 3}$ & $\mathbf{2 0 0 2}$ & $\mathbf{2 0 0 1}$ \\
Technologies de l'information & 468000 & 457000 & 459000 & 474000 & 492000 \\
(évol. / année précédente) & $(+2,4 \%)$ & $(-0,4 \%)$ & $(-3,2 \%)$ & $(-3,7 \%)$ & $(+0,4 \%)$ \\
Télécommunications & 288000 & 289000 & 294000 & 307000 & 327000 \\
(évol. / année précédente) & $(-0,3 \%)$ & $(-1,7 \%)$ & $(-4,2 \%)$ & $(-6,1 \%)$ & $(-0,9 \%)$ \\
Total & 756000 & 746000 & 753000 & 781000 & 819000 \\
& $(+1,3 \%)$ & $(-0,9 \%)$ & $(-3,6 \%)$ & $(-4,1 \%)$ & $(-0,1 \%)$ \\
\hline
\end{tabular}

Source des données : TNS Infratest Business Intelligence/BITKOM, mai 2005. *) Prévisions.

Après plusieurs années de stagnation, les investissements dans les TIC ont eux aussi repris l'an dernier, moins toutefois en Allemagne $(+2,5 \%$ ) qu'en moyenne mondiale $(+4 \%$; BMBF). Le ministère fédéral de la Recherche explique cette faiblesse comparative par la faible propension générale des entreprises à investir dans les biens d'équipement, une tendance conjoncturelle persistante. Et il rappelle, dans son rapport 2005 sur la compétitivité technologique de l'Allemagne, ce qui est la principale faiblesse de l'économie de l'information outreRhin : "plus nettement que dans d'autres pays, les compétences liées aux TIC se concentrent sur les applications industrielles ». Autrement dit : ce sont l'automobile et la construction mécanique qui tirent l'économie des TIC. Dans ce domaine aussi, la force de l'Allemagne réside plus dans les technologies incrémentales (en l'occurrence, l'électrotechnique) que dans les technologies de pointe. On peut observer une triple tendance générale à la concentration des activités de R\&D (2,5\% du PIB allemand) depuis quelques années : la R\&D, dans les TIC ou ailleurs, est le fait des grandes entreprises exposées à la concurrence et à la course à l'innovation mondiales, qui recentrent leurs activités sur quelques technologies de pointe (sans négliger les technologies moyennes). La restructuration progressive des TIC depuis le dot-com-crash reflète ainsi l'accélération de la globalisation des activités comme la spécialisation mondiale d'une économie allemande exportatrice, mais marquée aussi par un déséquilibre sectoriel croissant.

Cela étant, la dépendance de l'automobile (mais aussi de la construction mécanique) comporte également des aspects positifs. L'automobile, gourmande en équipements et services électroniques, qu'il s'agisse de rationaliser la production, d'accélérer l'innovation-produit, de maîtriser la logistique ou d'équiper les véhicules, crée une très forte demande de solutions informatiques, tirant l'innovation notamment dans les secteurs de la construction d'installations et de la robotique. Dans l'industrie automobile, $80 \%$ des inventions sont mises en $œ u v r e$ par ordinateur, autrement dit : sont tributaires d'un logiciel. La part est de $60 \%$ dans l'industrie électrotechnique. Dans la construction mécanique, selon sa fédération VDMA, l'automatisation et la robotique enregistrent une forte croissance : leur CA était de 6,9 milliards $€$ en 2004, en hausse de $5 \%$ par rapport à 2003. Selon la fédération de l'électrotechnique et de l'électronique, ZVEI, l'ensemble de la branche des technologies de l'automatisation réalise un CA de quelque 30 milliards $€$, plaçant l'Allemagne au premier rang mondial.

Le marché du logiciel et des inventions mises en œuvre par ordinateur bénéficie d'une forte demande nationale et mondiale. Certes, il faut ici distinguer 
entre les logiciels et progiciels 'standard' pour la gestion de données (du classique traitement de texte aux solutions EDI en passant par le CAD) d'un côté, et ceux qui sont destinés à piloter des installations et machines ou à en assurer le fonctionnement, de l'autre. Dans la première catégorie, un segment de masse, les entreprises américaines dominent le marché mondial en volume. Seules quelques sociétés allemandes comme SAP peuvent encore rivaliser avec elles, mais pour combien de temps ? La véritable force du marché allemand du software est ailleurs : d'une part dans le développement de solutions individualisées, tel que le proposent SAP, IDS Scheer ou Software AG. En réponse à une demande croissante de solutions sur mesure, dont les programmes de gestion de l'information qui booment avec l'essor de l'intelligence économique, les SII allemandes ont commencé à se transformer en solution-provider proposant des modules de systèmes intégrés. Ce faisant, elles répondent aussi (comme leurs homologues des autres Etats de I'UE, d'ailleurs) aux besoins générés par la structure encore très cloisonnée à la fois du marché communautaire comme des infrastructures de communication et solutions choisies par les entreprises, rappelle une étude de la Deutsche Bank.

\section{Brevets des inventions par ordinateur : un régime favorable aux PMA allemandes}

Si les fédérations ZVEI et BDI (le président du ZVEI est également vice-président du BDI) regrettent, dans un communiqué conjoint, que le Parlement européen ait rejeté, le 6 juillet 2005, le projet de directive sur la brevetabilité des inventions par ordinateur, c'est au nom d'une " protection efficace de la propriété intellectuelle... indispensable à la compétitivité $d u$ site high tech allemand». Mais, ajoutent-elles : "il vaut mieux le statu quo qu'une directive qui nous serait gravement dommageable en réduisant le degré de protection actuel ». En attendant la révision du projet de directive, c'est la pratique de l'Office européen des Brevets qui prévaut. Celui-ci accepte généralement de breveter les inventions par ordinateur ; à l'inverse des logiciels de base qui sont protégés par le seul copyright, donc disponibles pour les autres utilisateurs à faible coût en comparaison d'un brevet.

Or cette situation convient parfaitement au Mittelstand. Particulièrement dans la branche des technologies de l'automatisation où se recrutent nombre de ces hidden champions allemands, leaders mondiaux dans leur domaine, comme Baumüller, Harting, IFM ou Pepper+Fuchs. D'une manière générale, le rejet du projet de directive en l'état pourrait bénéficier aux PME de l'électronique et de l'électrotechnique : les deux tiers des entreprises du secteur ne déposent qu'un seul brevet ; ce sont des PME. Une limitation de la brevetabilité des inventions par ordinateur aurait mis en danger leur rentabilité et, par-delà, la compétitivité d'activités certes diffuses, mais essentielles pour le site de production allemand.

En réalité, la fédération ZVEI et son homologue européenne, tout comme la fédération de l'économie de l'information, des télécommunications et des nouveaux médias (BITKOM), se félicitent du rejet d'un texte qui aurait privé l'Europe d'un avantage compétitif sur les USA et le Japon qui, eux, peuvent protéger ces inventions en les brevetant. En l'absence d'un accord sur le brevet européen (qui implique qu'on tranche la question de la définition de la nature de l'invention), il est vrai que le statu quo proroge un cadre porteur pour les activités de R\&D des PME, ce qui, sans que cela soit intentionnel, va dans le sens d'une des priorités de la première comme de la seconde étape (i2010) de la stratégie de Lisbonne : la dynamisation des PME et leur intégration dans les réseaux européens de R\&D, dont ceux relatifs aux TIC.

La force du software allemand réside d'autre part dans le segment des mesures et asservissement, de l'automatisation comme de l'électronique embarquée. $98 \%$ des micro-processeurs sont aujourd'hui utilisés dans les installations industrielles, les véhicules, les produits bruns (de la hifi aux téléphones portables) ou blancs (comme les machines à laver). Software et hardware sont indissociables. Cette tendance mondiale, qui requiert au niveau de la conception comme de la production une grande proximité entre industrie et services et qui se nourrit d'améliorations de proche en proche, au plus près de la demande du marché, constitue un solide avantage compétitif pour l'Allemagne. Les branches phares de l'industrie - automobile, construction mécanique, sans oublier l'électrotechnique (voir le portrait de Siemens dans REA 70/05) - tirent leurs performances à l'export justement de ce qu'elles intègrent produit et services afférents. L'industrie allemande consacre les deux tiers de ses dépenses de R\&D aux logiciels et inventions mises en œuvre par ordinateur, rappellent la fédération de l'industrie BDI (essentiellement des PME) et la fédération ZVEI. C'est de la sorte que les branches-phares diffusent dans le tissu des activités, soutenant l'innovation des PME. L'étroit lien structurel industrie-services dynamise ainsi en retour l'innovation incrémentale sur le sol allemand : les moyennes et hautes technologies dont l'industrie a besoin pour asseoir ses parts de marché mondiales sont surtout le fait des PME.

2/3 des dépenses industrielles de $R \& D$ pour les inventions par ordinateur 
Software : une part de marché mondiale de $8 \%$

e-commerce : $30 \%$ du marché européen

$44 \%$ des entreprises règlent leurs factures en ligne

L'outil Internet concurrence I'ED
Dans un contexte mondial et européen où se dégage un fort potentiel de croissance pour l'ensemble des TIC (selon la Commission européenne, les TIC représentent $40 \%$ de la croissance de la productivité et $25 \%$ de la croissance du PIB de l'UE), l'Allemagne dispose de sérieux atouts. Sa part de marché mondiale dans le software ( $8 \%$ ) ne représente certes que le cinquième des USA, mais la place en tête des Etats membres, avant la Grande-Bretagne (7\%; 2004). Si l'Allemagne a vu ses parts de marché reculer ces dernières années, au profit de la Grande-Bretagne et des pays est-européens, elle n'en bénéficie pas moins toujours d'un solide effet de taille. En outre, selon la Deutsche Bank, la stagnation du marché du logiciel a pris fin l'an passé. Les SIl allemandes se sont repositionnées, et bénéficient d'un environnement conjoncturel plus porteur: depuis le recul de la demande de solutions standard qui avait culminé avec le passage à l'an 2000, la demande se diversifie. Outre-Rhin, les entrées en commande dans l'industrie ont en outre redémarré au début 2005, et dans le cadre du salon Cebit de 2005, les entreprises ont fait part de leur intention d'investir pour moderniser leurs équipements informatiques, explique par exemple l'institut ZEW (Branchenreport $\mathrm{n}^{\circ} 1$, avril 2005).

Au niveau des applications des TIC, il est un segment où l'Allemagne se caractérise par un dynamisme patent : celui de l'e-commerce, dont le CA s'élevait à 203 milliards $€$ en 2004 (BITKOM/EITO). Avec $30 \%$ du marché (le double de la France, au troisième rang), l'Allemagne est le leader européen, avant la Grande Bretagne (18\%). Par types d'activités: le seul commerce en ligne (B2C) représentait 4,4\% du PIB en 2003, nettement plus qu'en moyenne européenne (3,5\%) ; cette part est passée à plus de $6 \%$ en 2004 (BMBF). Pourtant, le B2C (CA : 22,7 milliards $€$ en 2004 ; BITKOM/EITO) pèse peu en comparaison du B2B qui non seulement se taille la part du lion avec 180,3 milliards $€$, mais connaît en outre une croissance annuelle de près de $40 \%$. Son CA devrait ainsi dépasser les 580 milliards $€$ en 2008.

Selon Destatis, qui détaille les pratiques des entreprises comme des particuliers en la matière, une entreprise sur deux se livrait au commerce électronique en 2003 : $52 \%$ des entreprises avaient accès à Internet. Les grandes entreprises (avec près de $66 \%$ ) sont bien entendu les plus férues de ce mode de communication. Sur le total des entreprises, $37 \%$ y recourent pour effectuer leurs commandes de biens et services (53\% des entreprises de plus de 250 salariés), et $44 \%$ pour le règlement de leurs factures. La vente en ligne pèse peu, en revanche, ne représentant que $1 \%$ du CA total des entreprises allemandes. Elle se concentre sur quelques secteurs, comme l'hôtellerie (29\% des transactions), la culture (18\%) ou le commerce de détail (18\%). A relever que, tous types confondus, l'écrasante majorité des transactions (84\%) s'effectue sur le territoire national (10\% seulement au sein du marché communautaire). Cela reflète les handicaps classiques des échanges transnationaux en ligne : une réglementation insuffisamment harmonisée, en matière de sécurité comme de droit commercial, se traduisant par la méfiance des acteurs.

Si les entreprises sont nombreuses à utiliser Internet pour leurs relations professionnelles (B2B), le Web se trouve concurrencé par les réseaux de communication tels l'intranet et l'extranet permettant des échanges directs et sécurisés. Ainsi, toujours en 2003, quelque 25000 entreprises recouraient systématiquement à ces réseaux et aux solutions de l'Electronic Data Interchange (EDI), effectuant de la sorte $27 \%$ de leurs dépenses pour achat de biens et services (et réalisant ainsi $13 \%$ de leur CA). Ces entreprises se recrutent pour l'essentiel dans le commerce $(46 \%)$ et l'industrie (32\%). Rapporté à l'ensemble des entreprises allemandes, la part de celles recourant à l'EDI est identique $(4 \%)$ dans ces deux secteurs. Très clairement, cette solution reste l'apanage des services financiers, préférée par $12 \%$ des établissements recensés outre-Rhin. Mais l'EDI est en perte de vitesse dans le secteur bancaire depuis le basculement à l'euro, qui a considérablement réduit les transactions de conversion. En revanche, depuis que les banques allemandes se sont lancées à la 
reconquête du retail (voir REA 66/04), elles misent massivement (à $85 \%$ ) sur l'outil Internet qui leur permet à la fois d'accroître leur visibilité et d'offrir à leur clientèle de masse une gamme complète de services. Cette stratégie est prisée par les Internautes allemands, qui sont $39 \%$ à pratiquer l'online banking.

TIC : taux d'équipement des ménages allemands *) (en \%)

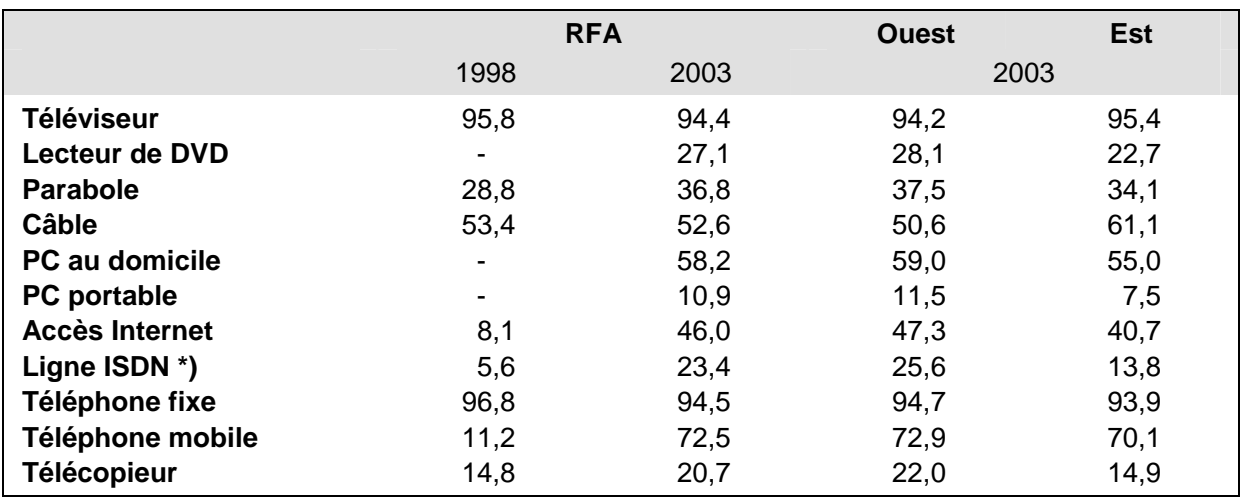

Source des données : Destatis, IKT in Deutschland, 2004. *) Part des foyers équipés d'au moins un de chacun de ces équipements. ${ }^{* *}$ ) L'appellation française est Numéris.

Les Allemands sont remarquablement équipés, à l'est comme à l'ouest. Les marchés des téléviseurs et des téléphones fixes ont atteint le stade de la saturation. II n'en est pas de même des téléphones portables : $78 \%$ des ménages en possèdent au moins un en 2004 (Destatis), et $22 \%$ en ont un permettant d'accéder à Internet. Selon l'autorité de régulation des télécommunications (RegTP), le nombre des abonnements mobiles dépasse depuis 2001 celui des fixes, avec respectivement 71,3 millions contre 54,6 millions en 2004. Pourtant, le taux de pénétration de la téléphonie mobile est plus faible outre-Rhin que dans les pays leaders que sont en Europe la Suède et l'Italie avec des taux de l'ordre de $100 \%$. Mais le coût des communications y est particulièrement élevé : selon l'institut ZEW, les tarifs moyens les moins chers oscillent entre $69,42 €$ (usage intensif) et $19 €$ (usage restreint). Ils sont supérieurs de respectivement $22 \%$ et $30 \%$ aux moyennes suédoises. Les raisons sont d'ordre commercial : pour fidéliser leurs clients (contrats exclusifs), la plupart des opérateurs subventionnent les portables ou vendent leurs cartes prepaid à des prix élevés, sans parler de la tarification dissuasive des appels des fixes vers les portables. Plus lourdes de conséquences sont les raisons structurelles: la faible tarification de la téléphonie fixe induite par le développement du dégroupage inhibe la substitution du portable au fixe. La concurrence entre les opérateurs du fixe est en effet particulièrement vive outre-Rhin, où les nouveaux entrants avaient une part de marché de $5 \%$ à la fin 2004. C'est la part la plus élevée en Europe, mais il faut dire que l'Allemagne a commencé à libéraliser le marché la première (dès 1998).

Les PC font, en moyenne du moins, partie aujourd'hui de l'équipement standard des ménages : $66 \%$ des foyers avaient un PC fixe en 2004. Et $60 \%$ avaient accès à Internet (Destatis ; moyenne européenne : $47 \%$ ), ce qui place l'Allemagne au second rang européen après le Danemark $(69 \%)$ et loin devant la France $(34 \%)$. Cela étant, si la fracture numérique tend à se réduire entre les générations et les catégories de revenus (la part des foyers Internet a crû de $30 \%$ depuis 2002), l'accès à Internet reste l'apanage des ménages jeunes et à hauts revenus ( $87 \%$ des foyers au revenu net mensuel supérieur à $3600 €)$.

Si la demande est forte, et si l'Allemagne est bien armée en matière d'équipements comme d'applications, elle souffre aujourd'hui d'un handicap alarmant dans le domaine des infrastructures à haut débit. Selon la fédération européenne des Télécommunications ECTA, le taux d'équipement allemand (8,9\%) figure parmi les plus bas d'Europe, du moins en comparaison d'un taux de $70 \%$ de foyers raccordables à un réseau câblé, un des plus élevés d'Europe. De
Le marché de la téléphonie mobile n'est pas encore saturé

$60 \%$ des ménages ont accès à Internet

Sous-développement du haut débit 
l'avis concordant de l'Institut ZEW, le problème réside dans le quasi-monopole du DSL $(97 \%$ des technologies de haut débit, contre $91 \%$ en France à la mi2004). C'est là le double héritage de la précocité de la numérisation et des choix effectués en matière d'architecture des réseaux. Un premier problème concerne pareillement les réseaux de téléphonie fixe et les réseaux câblés de distribution audiovisuelle : la multitude des acteurs au niveau de la boucle locale, en l'occurrence, du segment menant du point de livraison à la prise chez le client. Ce segment, réservé à l'artisanat local, donc au secteur privé, est géré par plusieurs milliers d'entreprises. La reconfiguration de l'accès client au téléphone haut débit est onéreuse, et l'adaptation du câble à l'interactivité requise par la téléphonie ou Internet (équipement en voies aller et retour) tient du casse-tête. C'est une des raisons pour lesquelles on s'intéresse de près, outreRhin, aux technologies sans fil (comme le WLAN), à la technologie des courants porteurs en ligne (power line) ou aux liaisons par satellite; mais ces deux dernières ne représentent encore que $1 \%$ du haut débit. Un second problème réside dans la trop faible concurrence parmi les opérateurs du DSL, où Deutsche Telekom domine largement ( $88 \%$ des abonnements). La solution consistera à aviver la concurrence en accélérant le dégroupage, l'interopérabilité, le resale (une pratique où l'institut ZEW cite en exemple l'Italie et la France) ou l'interconnexion des réseaux. Mais cela implique la convergence des régimes de l'audiovisuel et des télécommunications, un assouplissement de la réglementation en matière de télécommunications et une politique plus ferme de la RegTP vis-à-vis de Deutsche Telekom. L'enjeu est de taille - aussi bien pour l'avenir du marché des applications professionnelles que pour celui de l'électronique grand public, en forte croissance $(+8,3 \%$ en 2004 pour un CA de 20 milliards € selon la GFU, la fédération du secteur).

REDUIRE LES FREINS A L'INVESTISSEMENT. Voilà la priorité générale qui se dégage du rapport du BMBF sur la compétitivité technologique de l'Allemagne. Elle détermine aussi le soutien au développement des TIC. Comme il s'agit des technologies-clefs par excellence dont l'effet-levier sur la compétitivité globale est incommensurable, les politiques ne peuvent qu'agir sur l'environnement général réservé aux activités. Et si le BMBF regrette la faiblesse allemande dans le domaine high tech, y compris dans les TIC, c'est moins pour définir des priorités de recherche et lancer une nouvelle politique d'innovation que pour inciter les acteurs (industriels, PME, centres de recherche, mais aussi acteurs de la formation, consommateurs) à mieux encore conjuguer leurs efforts pour développer leur portefeuille de R\&D en l'élargissant vers le haut tout en entretenant un climat propice à l'innovation. "L'utilisation des TIC a un effet bénéfique sur les performances des entreprises lorsqu'elle est associée aux compétences et à une organisation complémentaires ", explique l'OCDE (2004). Vu sous cet angle, le potentiel allemand est énorme.

\section{Indications bibliographiques:}

BITKOM, Innovationen für Wachstum und Beschäftigung. Das 10-Punkte-Programm der ITK-Wirtschaft 2005, Berlin, 2005 (www.bitkom.org)

BMBF, Bericht zur technologischen Leistungsfähigkeit Deutschlands 2005 (www.bmbf.de) BouRGEOIS I., "Comment les PME allemandes passent-elles au numérique? ", in REA $n^{\circ} 61 / 03$, et « Les NTIC ", in REA 42/99"

DESTATIS, IKT in Deutschland. Informations- und Kommunikationstechnologien 1995-2003. Computer, Internet und mehr, Wiesbaden, 2004, et Informationstechnologie in Unternehmen und Haushalten 2004, Wiesbaden, 2005

OCDE, Perspectives des technologies de l'information, Principales conclusions, Paris, 2004

PAUTRAT M.-H., «E-gouvernement : l'impact du programme BundOnline 2005 », in REA 61/03

"Software-Häuser : Wandel vom Produkt-Hersteller zum Solution-Provider », Deutsche Bank Research, e-conomics, $\mathrm{n}^{\circ} 50$ (17-03-05),

TNS INFRATEST, Monitoring Informationswirtschaft. 8. Faktenbericht, 5. Trendbericht im Auftrag des BMWA, Munich, avril 2005 (www.bmwa.bund.de)

ZEW, Benchmark „Internationale Telekommunikationsmärkte“ im Auftrag des BMWA, Mannheim, 2005 (www.bmwa.bund.de) 